Aim of the study: The presence of BRCA germline mutations in patients with ovarian cancer has been shown to have predictive and prognostic significance, including increased platinum-sensitivity. The aim of the study was to evaluate if patients with BRCA1-associated ovarian cancer have more treatment related adverse events and, if so, does it have impact on chemotherapy outcomes.

Material and methods: We conducted a retrospective analysis of medical records of 172 patients with newly diagnosed epithelial ovarian cancer, treated in Maria Skłodowska-Curie Memorial Cancer Center and Institute of Oncology, Gliwice Branch between 2007 and 2013. Ninety-six of these patients have known BRCA mutation status - 21 patients were BRCA1(+) and 75 BRCA1(-). Analysed treatment related adverse events (AE's) were: haematological toxicity, nausea/vomiting, neuropathy and mucositis.

Results: Grade 3-4 haematological AE's were significantly more common among BRCA $1(+)$ patients $(\mathrm{OR}=3.86$; 95\% Cl: $1.14-13.23 ; p=0.02)$. There was no association between BRCA1 mutation status and neuropathy $(p=$ 0.73 ) or nausea/vomiting ( $p=0.91$ ). Occurrence of above mentioned AE's has no significant association with PFS ( $p=0.75,0.64,0.97$ respectively) and OS ( $p=0.64,0.69,0.73$ respectively). Conclusions: Among patients with BRCA1-associated epithelial ovarian cancer we observed significantly more grade 3-4 haematological complications after chemotherapy. However, occurrence of AE's did not correlate with better outcomes in this subgroup.

Key words: BRCA1, ovarian cancer, adverse events.

Contemp Oncol (Pozn) 2016; 20 (5): 381-384 DOI: $10.5114 /$ wo.2016.64597

\section{Treatment related toxicity in BRCA1-associated epithelial ovarian cancer - is DNA repairing impairment associated with more adverse events?}

\author{
Agnieszka Badora-Rybicka ${ }^{1}$, Magdalena Budryk², Elżbieta Nowara' ${ }^{1}$, \\ Danuta Starzyczny-Stota ${ }^{1}$
}

\author{
${ }^{1}$ Clinical and Experimental Oncology Department, Maria Skłodowska-Curie Memorial \\ Cancer Center and Institute of Oncology, Gliwice Branch, Poland \\ ${ }^{2}$ Genetic Outpatient Clinic, Maria Skłodowska-Curie Memorial Cancer Center and \\ Institute of Oncology, Gliwice Branch, Poland
}

\section{Introduction}

BRCA1 or BRCA2 germline mutation carriers account for $10-15 \%$ of epithelial ovarian cancer cases [1-3]. BRCA-associated ovarian cancers are usually of serous histology and, in comparison to sporadic ones, of higher grade, more advanced and diagnosed at a younger age [4].

BRCA1 and BRCA2 genes are considered tumor suppressor, since they play important role in the maintenance of genomic stability and cell growth [5]. The products of these genes - BRCA1 and BRCA2 proteins - are involved in the repair of DNA double-strand breaks via the homologous recombination pathway. This mechanism is believed to be responsible for increased chemo-sensitivity, which results in better treatment outcomes [6, 7]. However, the impact of BRCA dysfunction on the prognosis of patients with epithelial ovarian cancer remains controversial and available analyses brought conflicting results $[8,9]$. The better prognosis of patients with BRCA germline mutations is explained by cells inability to repair double-strand DNA breaks caused by platinum based chemotherapy [10].

BRCA germline mutations result in deficiency in repairing double-strand DNA breaks caused by platinum agents in every cell of the body. The aim of the study was to examine if patients with BRCA1-associated epithelial ovarian cancer have more treatment related adverse events and, if so, does it have impact on chemotherapy outcomes.

\section{Material and methods}

We conducted a retrospective analysis of an unselected population of patients treated in Maria Skłodowska-Curie Memorial Cancer Center and Institute of Oncology, Gliwice Branch between 2007 and 2013. Pathological stage of the disease was categorized according to International Federation of Gynaecology and Obstetrics (FIGO) 2009. The date of diagnosis was defined as the date of initial surgery. Response to chemotherapy was determined by computed tomography (CT) scan after completion of systemic treatment according to RECIST criteria 1.0. Recurrence/progression was diagnosed by $\mathrm{CT}$, regardless of Ca-125 rise. Treatment related adverse events (AE's) were: haematological toxicity, nausea/vomiting, neuropathy and mucositis. Grade of AE's was determined using Common Terminology Criteria for Adverse Events (CTCAE). Platinum-resistant disease was defined by recurrence at $\leq 6$ months after completion of chemotherapy. 
Overall survival (OS) was calculated from the date of diagnosis to the date of patient's death of any cause or last seen alive. Progression free survival (PFS) was calculated from the date of diagnosis the date of disease recurrence or patient's death. We used exact Fisher test and $\chi^{2}$ test to compare BRCA1(+) and BRCA1(-) subgroups. The relationship between the occurrence of adverse events and treatment outcomes was analysed using the U Mann-Whitney and $\chi^{2}$ tests.

\section{Results}

One hundred and seventy-two 172 patients were treated for ovarian cancer at Maria Skłodowska-Curie Memorial Cancer Centre Gliwice Branch between 2007 and 2013. 96 of these patients have known BRCA mutation status (21 patients were BRCA1 positive and 75 - BRCA1 negative) and were included in our analysis. We did not note any BRCA2 mutation.

Median age in both subgroups was 52 years. Baseline performance status of patients was 0 (89 patients, 93\%) or 1 (7 patients, $7 \%$ ), with no severe comorbidities. Clinical and pathological characteristics of patients are detailed in Table 1.

Baseline Ca-125 level was significantly higher among BRCA1 $(+)$ woman $(p=0.04)$. We did not note significant differences in other analyzed parameters between BRCA1(+) and BRCA1(-) patients.

All patients received combined platinum-taxane chemotherapy (carboplatin AUC 5 and paclitaxel $175 \mathrm{mg} / \mathrm{m}^{2}$, every 21 days, intravenously). The number of cycles was 3-9, most patients received 6 cycles of chemotherapy (81 patients, $84 \%$ ). We used ondansetron $8 \mathrm{mg}$ (once or twice daily) and dexamethasone $8 \mathrm{mg}$ in prevention of chemotherapy-induced nausea and vomiting. In case of insufficient antiemetic effect metoclopramide and/or thiethylperazine were applied. We did not use haematpoietic growth factors in any patient. For persistent neuropathy symptoms $\alpha$ lipoic acid $600 \mathrm{mg}$ once daily was applied. In case of oral candidiasis we used locally nystatin or oral fluconazole $100 \mathrm{mg}$ once daily.

We did not note differences in response to chemotherapy (complete remission, partial response, stable disease, progression) between $\mathrm{BRCA1}(+)$ and $\mathrm{BRCA1}(-)$ patients $(\mathrm{OR}=1.71 ; 95 \% \mathrm{Cl}: 0.64-4.56 ; p=0.28)$. Median PFS was 28.6 (8.4-69.2) months among BRCA1(+) patients and 22.2 (6.3-79.7) months in BRCA1 $(-)(p=0.45)$. Median OS was 39.6 (16.3-79.8) months for BRCA1(+) patients and 38.2 (7.2-93.9) months for BRCA1 $(-)(p=0.62)$.

The presence of BRCA1 mutation was not associated with more frequent occurrence of haematological toxicities of any grade $(\mathrm{OR}=2.11 ; 95 \% \mathrm{Cl}: 0.79-5.64 ; p=0.13)$, however, grade $3-4$ haematological AE's were significantly more common among BRCA1 $(+)$ patients $(\mathrm{OR}=3.86$; $95 \%$ $\mathrm{Cl}: 1.14-13.23 ; p=0.02)$. Neuropathy of any grade and neuropathy grade 3-4 were noted in comparable number of cases among both groups $(\mathrm{OR}=1.19 ; 95 \% \mathrm{Cl}$ : 0.45-3.17; $p=0.73$ and $\mathrm{OR}=0.89 ; 95 \% \mathrm{Cl}: 0.09-8.39 ; p=0.92$, respectively). Nausea and vomiting of any grade occurred comparable in both groups $(\mathrm{OR}=0.94 ; 95 \% \mathrm{Cl}$ : 0.34-2.62; $p=0.91)$. The number of nausea/vomiting grade $3-4$ cases (2 in BRCA1(-) and 0 in BRCA1(+) patients) was too small to perform statistical analysis. The number of patients with mucositis was also too small to conduct credible analysis ( 1 case in $\mathrm{BRCA}(-)$ and 0 in $\mathrm{BRCA1}(+)$ patients, with no case of grade 3-4 mucositis). These results are summarized in Table 2.

We did not notice more drugs dose reductions among hereditary ovarian cancer patients $(\mathrm{OR}=3.29 ; 95 \% \mathrm{Cl}$ : $0.8-13.59 ; p=0.09$ ).

Table 1. Clinical and pathological characteristics of patients

\begin{tabular}{|c|c|c|c|}
\hline Parameter & $\begin{array}{c}\text { BRCA1(+) } \\
n=21\end{array}$ & $\begin{array}{c}\text { BRCA1(-) } \\
n=75\end{array}$ & $p$ \\
\hline Median age [years] & $52(37-65)$ & $52(23-73)$ & \\
\hline $\begin{array}{l}\text { FIGO stage } \\
\text { I } \\
\text { II } \\
\text { III } \\
\text { IV }\end{array}$ & $\begin{array}{c}3(14.3 \%) \\
1(4.8 \%) \\
11(52.4 \%) \\
6(28.5 \%)\end{array}$ & $\begin{array}{c}16(21.3 \%) \\
9(12 \%) \\
44(58.7 \%) \\
6(8 \%)\end{array}$ & 0.1 \\
\hline $\begin{array}{l}\text { Pathology } \\
\text { Serous } \\
\text { Mucinous } \\
\text { Endometrioid } \\
\text { Clear cell } \\
\text { Others }\end{array}$ & $\begin{array}{c}17(80.9 \%) \\
1(4.8 \%) \\
2(9.5 \%) \\
0 \\
1(4.8 \%)\end{array}$ & $\begin{array}{c}44(58.7 \%) \\
7(9.3 \%) \\
9(12 \%) \\
5(6.7 \%) \\
10(13.3 \%)\end{array}$ & 0.53 \\
\hline Optimal debulking & $9(42.8 \%)$ & $50(65.3 \%)$ & 0.08 \\
\hline Secondary cytoreduction & $8(66.7 \%)$ & $25(33.3 \%)$ & 0.88 \\
\hline Median baseline Ca-125 level [IU/I] & 265 & 32.65 & 0.04 \\
\hline Disease progression after $1^{\text {st }}$ line chemotherapy & $13(61.9 \%)$ & $41(54.7 \%)$ & 0.73 \\
\hline $\begin{array}{l}\text { Platinum sensitivity } \\
\text { Sensitive } \\
\text { Resistant }\end{array}$ & $\begin{array}{c}19(90.5 \%) \\
2(9.5 \%)\end{array}$ & $\begin{array}{l}64 \text { (85.3\%) } \\
11(14.6 \%)\end{array}$ & 0.73 \\
\hline
\end{tabular}


Table 2. The relationship between chemotherapy related AE's and BRCA1 mutation status

$\begin{array}{lccc}\text { Parameter } & \text { BRCA1(+) } & \text { BRCA (-) } & p \\ \text { Haematological AE's } & & & \\ \text { Any grade } & 12(57 \%) & 29(38.7 \%) & 0.13 \\ \text { Grade 3-4 } & 6(28.5 \%) & 7(9.3 \%) & \\ \text { Neuropathy } & & & \\ \text { Any grade } & 9(42.9 \%) & 29(38.7 \%) & 0.73 \\ \text { Grade 3-4 } & 1(4.8 \%) & 4(5.3 \%) & 0.92 \\ \text { Nausea/vomiting } & 7(33.3 \%) & 26(34.7 \%) & 0.94 \\ \text { Any grade } & 0 & 2(2.7 \%) & 0.91\end{array}$

Table 3. The relationship between chemotherapy related AE's and treatment outcomes in BRCA1(+) patients

\begin{tabular}{|c|c|c|c|c|}
\hline Parameter & Median PFS (months) & $p$ & Median OS (months) & $p$ \\
\hline $\begin{array}{l}\text { Haematolo } \\
\text { Yes } \\
\text { No }\end{array}$ & $\begin{array}{l}24.4 \\
28.9\end{array}$ & 0.75 & $\begin{array}{l}37.7 \\
39.6\end{array}$ & 0.64 \\
\hline $\begin{array}{l}\text { Neuropathy } \\
\text { Yes } \\
\text { No }\end{array}$ & $\begin{array}{l}23.7 \\
28.9\end{array}$ & 0.64 & $\begin{array}{l}39.6 \\
37.7\end{array}$ & 0.69 \\
\hline $\begin{array}{l}\text { Nausea/vor } \\
\text { Yes } \\
\text { No }\end{array}$ & $\begin{array}{l}29.3 \\
26.5\end{array}$ & 0.97 & $\begin{array}{l}39.6 \\
37.7\end{array}$ & 0.73 \\
\hline
\end{tabular}

Among $\operatorname{BRCA} 1(+)$ patients occurrence of any of above mentioned adverse events was not predictive for response to chemotherapy. In this subgroup haematological toxicities were not predictive for PFS $(p=0.75)$ and OS ( $p=$ $0.64)$, as well as neuropathy ( $p=0.64$ and $p=0.69$ respectively) and nausea/vomiting ( $p=0.97$ and $p=0.73$ respectively) (Table 3 ).

\section{Discussion}

Evidence exists that ovarian cancer patients carrying germline BRCA mutations have an improved prognosis in comparison to sporadic cases [9-11]. BRCA-associated ovarian carcinomas are more aggressive but show higher susceptibility to platinum-salts and other DNA-damaging agents [10-15]. This phenomenon is explained by the fact that deficiency of BRCA1 or BRCA2 function leads to higher degree of chromosome instability and triggers alternative, less effective pathways to repair double-strand DNA breaks, resulting in accumulation of mutation events [15]. This mechanism is believed to be responsible for better response to platinum-based chemotherapy, but it may be also associated with higher toxicity of these agents $[6,7]$.

Data regarding differences in prognosis between BRCA1 and BRCA2 mutation related ovarian cancer are conflicting. Taken together, available studies confirm a significantly improved survival in BRCA2 mutation carriers in comparison to sporadic ovarian cancer patients, while this advantage for BRCA1 mutation carriers seems to be smaller $[8,10]$. It might be explained by the fact that BRCA1 and BRCA2 work at different stage of DNA repair process. BRCA2 is believed to be the RAD51 protein regulator. This protein is required for homologous recombination pathway functioning. According to this hypothesis, BRCA2 is a mediator of the core mechanism of homologous recombination, while BRCA1 is more "universal" protein. We included only patients with BRCA1 mutation in our analysis, since we did not note any case of BRCA2 gene mutation. We observed significantly higher median baseline Ca-125 level (265 vs. $32.65 \mathrm{IU} / \mathrm{l}, p$ $=0.04)$ and numerically more FIGO stage IV cases $(28.5 \%$ vs. $8 \%$ ) among BRCA1(+) patients. Despite this, we did not note worse survival outcomes in this group in comparison to sporadic cases. These observations are in concordance with above mentioned literature data [8-12]. However, interpretation of our results should be careful due to small sample size.

Our study is retrospective and thus has some limitations. Besides small sample size, not all patients treated in the analysed time period were tested for BRCA mutation, so a selection bias might occur. Patients at younger age or/ and with family history of breast or ovarian cancer cases are more likely to be referring to genetic testing. Not all patients were operated on at the same centre and we can therefore not be certain of uniform surgical quality. Moreover, reporting the occurrence of chemotherapy related AE's might be biased due to retrospective nature of our study.

In summary, our data suggest that BRCA1(+) woman receiving platinum-based chemotherapy for epithelial ovarian cancer are more likely to experience grade 3-4 haematological adverse events $(p=0.02)$. This may result from higher sensitivity of their body cells to platinum agents. However, among BRCA1(+) patients occurrence of haematological AE's or any other AE's did not translate into better treatment outcomes. Our findings warrant further investigation in larger, prospective studies. 
The authors declare no conflict of interest.

\section{References}

1. Risch HA, McLaughlin JR, Cole DE, et al. Prevalence and penetrance of germline BRCA1 and BRCA2 mutations in a population series of 649 women with ovarian cancer. Am J Hum Genet 2001; 68: 700-10.

2. Frank TS, Deffenbaugh AM, Reid JE, et al. Clinical characteristics of individuals with germline mutations in BRCA1 and BRCA2: analysis of 10,000 individuals. J Clin Oncol 2002; 20: 1480-90.

3. Beristain E, Martínez-Bouzas C, Guerra I, et al. Differences in the frequency and distribution of BRCA1 and BRCA2 mutations in breast/ovarian cancer cases from the Basque country with respect to the Spanish population: implications for genetic counselling. Breast Cancer Res Treat 2007; 106: 255-62.

4. Boyd J, Sonoda Y, Federici MG, et al. Clinicopathologic features of BRCA-linked and sporadic ovarian cancer. JAMA. 2000; 283: 2260-5.

5. Yoshida K, Miki Y. Role of BRCA1 and BRCA2 as regulators of DNA repair, transcription, and cell cycle in response to DNA damage. Cancer Science 2004; 95: 866-71.

6. Cass I, Baldwin RL, Varkey T, Moslehi R, Narod SA, Karlan BY. Improved survival in women with BRCAassociated ovarian carcinoma. Cancer 2003; 97: 2187-95.

7. Tan DS, Rothermundt C, Thomas K, et al. "BRCAness" syndrome in ovarian cancer: A casecontrol study describing the clinical features and outcome of patients with epithelial ovarian cancer associated with BRCA1 and BRCA2 mutations. J Clin Oncol 2008; 26: 5530-6.

8. Sun C, Li N, Ding D, Weng D, Meng L, Chen G, Ma D. The role of BRCA status on the prognosis of patients with epithelial ovarian cancer: a systematic review of the literature with a meta-analysis. PLoS One 2014; 9: e95285.

9. Bolton KL, Chenevix-Trench G, Goh C, et al. Association between BRCA1 and BRCA2 mutations and survival in women with invasive epithelial ovarian cancer. JAMA 2012; 307: 382-90.

10. Gallagher DJ, Konner JA, Bell-McGuinn KM, et al. Survival in epithelial ovarian cancer: a multivariate analysis incorporating BRCA mutation status and platinum sensitivity. Ann Oncol 2011; 22: 1127-32.

11. Chen P, Huhtinen K, Kaipio K, et al. Identification of prognostic groups in high-grade serous ovarian cancer treated with platinum-taxane chemotherapy. Cancer Res 2015; 75: 2987-98.

12. Safra T, Borgato L, Nicoletto MO, et al. BRCA mutation status and determinant of outcome in women with recurrent epithelial ovarian cancer treated with pegylated liposomal doxorubicin. Mol Cancer Thera 2011; 10: 2000-7.

13. Kaye SB, Lubinski J, Matulonis U, et al. Phase II, open-label, randomized, multicenter study comparing the efficacy and safety of olaparib, a poly (ADP-ribose) polymerase inhibitor, and pegylated liposomal doxorubicin in patients with BRCA1 or BRCA2 mutations and recurrent ovarian cancer. J Clin Oncol 2012; 30: 372-9.

14. Kwa M, Edwards S, Downey A, Reich E, Wallach R, Curtin J, Muggia F. Ovarian cancer in BRCA mutation carriers: improved outcome after intraperitoneal (IP) cisplatin. Ann Surg Oncol 2014; 21: 1468-73.

15. Banerjee S, Kaye S. PARP inhibitors in BRCA genemutated ovarian cancer and beyond. Curr Oncol Rep 2011; 13: 442-9.

\section{Address for correspondence}

\section{Agnieszka Badora-Rybicka}

Clinical and Experimental Oncology Department

Maria Skłodowska-Curie Memorial Cancer

Center and Institute of Oncology

Gliwice Branch

Wybrzeże Armii Krajowej 15

44-101 Gliwice, Poland

e-mail: agnieszka.k.badora@gmail.com

Submitted: 3.01 .2016

Accepted: $\quad 14.03 .2016$ 